\title{
Antiresonance scattering at defect levels in the quantum conductance of a one-dimensional system
}

\author{
X. R. Wang, ${ }^{1}$ Yupeng Wang, ${ }^{2}$ and Z. Z. Sun ${ }^{1}$ \\ ${ }^{1}$ Physics Department, The Hong Kong University of Science and Technology, Clear Water Bay, Hong Kong, People's Republic of China \\ ${ }^{2}$ Institute of Physics \& Center for Condensed Matter Physics, Chinese Academy of Sciences, Beijing 100080, \\ People's Republic of China
}

(Received 9 January 2002; published 29 April 2002)

\begin{abstract}
For the ballistic quantum transport, the conductance of each channel is quantized to a value of $2 e^{2} / h$. In the presence of defects, electrons will be scattered such that the conductance will deviate from the values of the quantized conductance. We show that an antiresonance scattering can occur when an extra defect level is introduced into a conduction band. At the antiresonance scattering, exactly one quantum conductance of a one-dimensional wire disappears, in good agreement with ab initio calculations. The conductance takes a nonzero value when the Fermi energy is away from the antiresonance scattering.
\end{abstract}

DOI: 10.1103/PhysRevB.65.193402

PACS number(s): 72.80.Rj, 72.10.Fk, 73.63.Nm

A large number of studies have been focused on electronic transport in quantum wires in recent years as we move into the era of nanoscience and nanotechnology. This is largely due to its academic interests and great importance in applications of the nanoscale electronic devices. Interesting phenomena, such as quantized conductance ${ }^{1,2}$ and 0.7 anomaly in conductance, ${ }^{3,4}$ were discovered. In this area, one of important subjects is the quantum transport properties of a system related to the motion of a single or a few electrons in a single or a few conducting channels. The conductance of a nanosystem with a few channels is best described by the Landauer formula, $G=\left(2 e^{2} / h\right) \operatorname{Tr}\left(t^{+} t\right)$, where $t$ is the transmission matrix. Thus, the conductance can be obtained from a microscopic quantum-mechanical calculation of transmission coefficients. An individual defect or impurity is expected to modify substantially the properties of such a system.

Among many systems, carbon nanotubes have received a particular attention because it is a new form (other than graphite, diamond, and fullerenes) of carbon, ${ }^{5}$ and because of great progress in carbon nanotube fabrications. Carbon nanotubes have very rich atomic and electronic structures. For the atomic structure, the tubes can be both single and multiple walls. The diameter of the tubes can vary within a single tube or from one tube to another. The nanotube structures can also be manipulated. For the electronic structure, a carbon nanotube can be either an insulator or a metal, depending on how the nanotube is wrapped up from a graphite sheet. Regarding the electronic transport, early experiments observed quantized electronic conductance ${ }^{6}$ in such tubes at room temperature. Recently, there are $a b$ initio calculations on the effects of impurities and local structural defects on the conductance of metallic carbon nanotubes. It was shown that the conductance is reduced by a quantum unit $\left(2 e^{2} / h\right)$ by a localized defect when the Fermi energy is at this defect level. However, the conductance is only reduced by a fractional of the quantum conductance unit when the Fermi energy is away from the defect level. Motivated by this discovery, we would like to investigate how a single local defect level affects conductance of a conducting channel.

There are two types of defects in a conduction band. One is that an atomic orbital deviates from its original form with- out changing the total number of orbitals. This may be achieved by substituting one atom by a foreign atom (of course, an electronic structure does not necessarily change in the same way as its atomic structure). The other is to add an extra orbital into the band by, for example, doping an impurity into a sample. We shall show that the two types of defects affect electronic transport in a quasi one-dimensional conduction band differently.

In order to consider the effects of an extra defect level on electronic-transport properties, we model the onedimensional metallic tube by a conventional one band tightbinding Hamiltonian on a chain. The Fermi energy will be restricted within the band. An extra defect state of energy $u$ is placed at site $i=0$. The nature of locality of the defect is modeled through a delta coupling of the defect state with conduction states at $i=0$. The Hamiltonian of this system can be written as

$$
\mathcal{H}=t \sum_{i} c_{i}^{\dagger} c_{i+1}+u d_{0}^{\dagger} d_{0}+v c_{0}^{\dagger} d_{0}+\text { c.c. }
$$

where $c_{i}^{\dagger}$ and $c_{i}$ are the creation and annihilation operators for an atomlike orbital centered at site $i . d_{0}^{\dagger}$ and $d_{0}$ are the creation and annihilation operators of the defect state at the origin. $v$ is the coupling coefficient between the localized defect state and band state at the origin. For simplicity, only the nearest-neighbor hopping is included in this model. In fact, the dynamical impurity problem described in the Hamiltonian could be generated effectively by a static impurity in a quantum wire. Assume that there is a static impurity potential around the origin of a wire. This impurity potential may create an extra impurity state $d(x, y)$ in the wire. The wave function of a conduction electron can be expressed as $\phi(x, y)$, which along the $y$ direction is dispersionless (here we consider only one subband). Therefore, the wave function $\phi(x, y)$ can be decomposed into $\phi(x, y)=\alpha c(x, y)$ $+\beta d(x, y)$, where $c(x, y)$ represent the conduction states of a pure wire, which itself can be decomposed into some local atomic orbits generated by creation operators $c_{i}^{\dagger}, \alpha$ and $\beta$ are two normalization constants with $|\alpha|^{2}+|\beta|^{2}=1$. Assume that the static impurity will only mix those nearest local atomic orbits of $c(x, y)$ with the impurity state, in the tight- 
binding representation, one obtains essentially the Hamiltonian (1). In the case that the static impurity does not create an extra impurity state, the same argument will lead to the Hamiltonian (5) studied later.

Without the defect, the eigenstates of the Hamiltonian (1) are the Bloch wave functions, and the eigenenergies of the Hamiltonian are $\epsilon=2 t \cos (k a)$, with $k=n \pi a / L, \quad n$ $=0,1,2, \ldots, N$, where $a$ is the lattice constant and $L$ is the length of the wire. This spectrum leads to an energy band of width $4 t$. When this band is partially filled, it forms the conduction band of the metallic wire. At zero temperature, there are two conducting channels at the Fermi energy. One propagates from the left to the right side, and the other in the opposite direction. The conductance should be $2 e^{2} / h$ in the ballistic region where a conduction electron does not experience any scattering. In the presence of a defect, the electron will be scattered by the defect, and the conductance should deviate from the quantized conductance unit according to the Landauer formula.

The central physical quantity is the transmission coefficient of an electron propagating from the left to the right sides of the chain through the defect. We assume that the electron initially has momentum $k$ and energy $\epsilon$ $=2 t \cos (k a)$ in the left hand side of the chain. In the tightbinding formalism the wave function has the following form

$$
\begin{aligned}
\phi= & \frac{1}{\sqrt{L}} \sum_{j=-\infty}^{-1}\left(e^{i k j}+R e^{-i k j}\right) c_{j}^{\dagger}|0\rangle+A c_{0}^{\dagger}|0\rangle+B d_{0}^{\dagger}|0\rangle \\
& +\frac{1}{\sqrt{L}} \sum_{j=1}^{\infty} T e^{i k j} c_{j}^{\dagger}|0\rangle,
\end{aligned}
$$

where $R$ and $T$ are the reflection and transmission coefficients, respectively. $A$ is the wave-function amplitude at the origin from the conduction state, and $B$ is that of the defect state. They can be obtained from equation $\mathcal{H} \phi=\epsilon \phi$. The transmission coefficient is given by

$$
T(\epsilon)=\frac{2 t(\epsilon-u) \sqrt{1-\left(\frac{\epsilon}{2 t}\right)^{2}}}{2 t(\epsilon-u) \sqrt{1-\left(\frac{\epsilon}{2 t}\right)^{2}}-v^{2} i} .
$$

Obviously, the transmission coefficient is unity when the coupling constant $v$ equals to zero. $v=0$ means that conduction band does not couple to the defect state. Thus a conduction electron will not experience any scattering. In order to have a better understanding of the transmission coefficient formula, we rewrite Eq. (3) for the weak coupling as

$$
T(\epsilon)=\sum_{n=0}^{\infty}\left(\frac{v^{2}}{2 t(\epsilon-u) \sqrt{1-\left(\frac{\epsilon}{2 t}\right)^{2}}} i\right)^{n} .
$$

Then, one can interpret Eq. (3) as the result of the sum of multiple-scattering process: an electron is virtually scattered from $c_{0}^{\dagger}|0\rangle$ state to $d^{\dagger}|0\rangle$ state and back to $c_{0}^{\dagger}|0\rangle$ state $n$ times with a strength of $i v^{2} /\left[2 t(\epsilon-u) \sqrt{1-(\epsilon / 2 t)^{2}}\right]$ in each time. It is useful to notice that $\pm 2 t$ are the edges of the conduction band. In fact, the above interpretation is exactly what the usual Green's-function formalism would give.

There are several nontrivial results in Eq. (3). First, an antiresonance scattering occurs when the electron energy is equal to $u$, the energy of the defect level. At the antiresonance, the transmission coefficient vanishes. In other words, the conducting channel is completely blocked by the defect when the Fermi energy is right at the defect energy level. Thus, the conductance of the wire is reduced by a quantum conductance unit, exactly what was observed in the ab initio calculations. ${ }^{7}$ It can be shown that the eigenfunction of Hamiltonian (1) for $\epsilon=u$ is

$$
\phi=\frac{2 i}{\sqrt{L}} \sum_{j=-\infty}^{-1} \sin (k j) c_{j}^{\dagger}|0\rangle-\frac{i}{\sqrt{L}} \sqrt{\left(\frac{2 t}{v}\right)^{2}-\left(\frac{u}{v}\right)^{2}} d_{0}^{\dagger}|0\rangle .
$$

This is a standing-wave-like state. The electron propagating from the left encounters the defect state, and then is completely reflected back to the left. The wave-function amplitude of the conduction state at the origin becomes zero, and part of incoming electron is trapped in the defect state. According to Eq. (3), the transmission coefficient also becomes zero at the band edge $\epsilon= \pm 2 t$. However, it can be shown that coefficients of both $A$ and $B$ in wave function (2) are zero in this case. Thus, no electron is trapped in the defect state. This is different from the previous case of the antiresonant scattering. This band-edge scattering effect is also consistent with early numerical result ${ }^{8}$ that an electron at the band edges is completely reflected back at a boundary of a narrow conductor and a wide contact. It reflects the fact that states at band edges are fragile, and are easily influenced by a perturbation. Another interesting result of Eq. (3) is that transmission coefficient approaches zero as the coupling strength $v$ between the defect state and conduction levels goes to infinity. This is, in some sense, due to the equivalence of infinite $v$ and the hard-wall condition where the wave-function amplitude should be zero.

The antiresonance scattering is due to the presence of an extra defect state. In order to show the importance of the extra defect level, we can examine another one impurity model. Consider the usual Anderson localization model with one impurity,

$$
\mathcal{H}=t \sum_{i} c_{i}^{\dagger} c_{i+1}+u c_{0}^{\dagger} c_{0}+\text { c.c. }
$$

which can describe a substituting impurity at the origin. The defect is due to the imperfection of periodicity at the origin, but the total number of orbitals does not change. Since there is only one level at the origin, it is impossible to have a resonance (antiresonance) scattering. The transmission coefficient of an electron with energy $\epsilon$ in the conduction band is given by 


$$
T(\epsilon)=\frac{2 t \sqrt{1-\left(\frac{\epsilon}{2 t}\right)^{2}}}{2 t \sqrt{1-\left(\frac{\epsilon}{2 t}\right)^{2}}-u i} .
$$

Clearly, there is no antiresonance for this model, but the band-edge effects are still present as expected.

Furthermore, we would like to demonstrate that the antiresonance scattering is very robust as long as an extra defect level exists in the conduction band. To do so, we generalize the Hamiltonian (1) to the case that the defect level couples also to conduction states at site $i= \pm 1$ besides the origin $(i=0)$. The Hamiltonian reads

$$
\mathcal{H}=t \sum_{i} c_{i}^{\dagger} c_{i+1}+\left(u d_{0}^{\dagger}+v c_{0}^{\dagger}+v_{1} c_{1}^{\dagger}+v_{1} c_{-1}^{\dagger}\right) d_{0}+\text { c.c. }
$$

where $v_{1}$ is the coupling constant between the defect state with the conduction states at $i= \pm 1$. Similarly, we can obtain the transmission coefficient $T(\epsilon)$

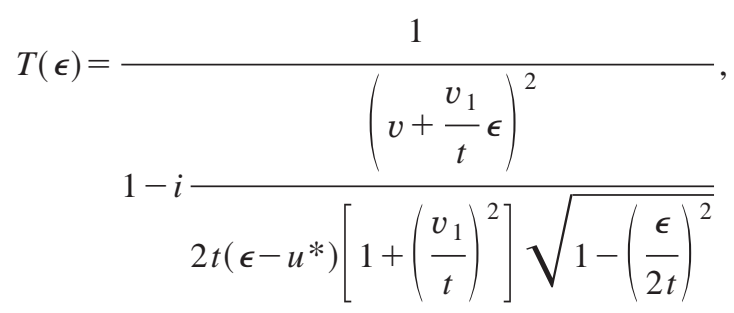

with normalized defect level $u^{*}=\left[u-\left(2 v v_{1} / t\right)\right] /[1$ $\left.+\left(v_{1} / t\right)^{2}\right]$. One can see, again, that an antiresonance scattering exists at the normalized defect level $u^{*}$. It recovers Eq. (3), $u^{*}=u$, when $v_{1}=0$, while the antiresonance occurs in the band center, $u^{*}=0$, when $v_{1} \rightarrow \infty$. It may be interesting to notice that Eq. (8) also contains a conventional resonance state at $\epsilon=-v t / v_{1}$, at which $T$ is unity.

In reality, a carbon nanotube can have more than one band. The defect level can then couple to many bands. In order to show that the antiresonance will not be affected by existence of many bands. We consider the following Hamiltonian for a two-band model,

$$
\begin{aligned}
\mathcal{H}= & t_{1} \sum_{i} c_{1, i}^{\dagger} c_{1, i+1}+t_{2} \sum_{i} c_{2, i}^{\dagger} c_{2, i+1}+u d_{0}^{\dagger} d_{0}+v_{1} c_{1,0}^{\dagger} d_{0} \\
& +v_{2} c_{2,0}^{\dagger} d_{0}+2\left(\left|t_{1}\right|+\left|t_{2}\right|+\frac{\Delta}{2}\right) \sum_{i} c_{2, i}^{\dagger} c_{2, i}+\text { c.c. }
\end{aligned}
$$

where $c_{\alpha, i}^{\dagger}$ and $c_{\alpha, i}(\alpha=1,2)$ are the creation and annihilation operators for an atomlike orbital centered at site $i$ for band $\alpha$. In the absence of extra defect level $d_{0}^{\dagger}|0\rangle$, this Hamiltonian supports two energy bands with band widths of $4\left|t_{1}\right|$ and $4\left|t_{2}\right|$, respectively. $\Delta$ is the energy gap between the two bands. The defect level couples to these two bands at the origin $(i=0)$ with $v_{1}$ and $v_{2}$ as the coupling coefficients, respectively. In the following analysis, we shall assume $\Delta$
$>0$. This two-band model can also be solved exactly. For $\epsilon$ $\leqslant 2\left|t_{1}\right|$, the electron transmission coefficient is given by

$$
T(\epsilon)=\frac{1}{1-i \frac{v_{1}^{2}}{2 t_{1}\left(\epsilon-u-\frac{v_{2}^{2}}{2 t_{2} \sinh \alpha}\right) \sqrt{1-\left(\frac{\epsilon}{2 t}\right)^{2}}}}
$$

where $\alpha=\cosh ^{-1}\left(2\left|t_{1}\right|+2\left|t_{2}\right|+\Delta-\epsilon / 2 t_{2}\right)$. It is easy to show that Eq. (10) becomes Eq. (3) when the coupling between the defect level and the second band is zero, i.e., $v_{2}=0$. Again, antiresonance occurs when the electron energy is at a particular level given by the equation $\epsilon=u+v_{2}^{2} / 2 t_{2} \sinh \alpha$.

The antiresonance is the physics of a quasi-onedimensional system when an extra defect level is introduced into the energy band. This phenomenon does not occur if a defect is introduced in such a way that the total number of electronic states in the band does not change, such as the Hamiltonian (5). We showed that this phenomenon is robust against the details of a microscopic Hamiltonian, whether the extra defect level couples to a single site [Hamiltonian (1)] or several sites [Hamiltonian (7)], or the defect couples to more than one band [Hamiltonian (9)]. This antiresonance can lead to localization of electronic states in one-dimensional systems. Imagine a pair of identical defect levels are introduced into a conduction band, an electron at antiresonance scattering with the two defect levels will be localized between the two defects since the electron will be fully scattered back and forth. When defect levels are randomly introduced into a system, then there is a probability of 1 to find a pair of levels of any given energy in the thermodynamic limit. Thus, one will expect all the states to be localized in one-dimensional system.

We would like to make a comparison between the antiresonance studied here and the famous Fano resonance. ${ }^{9}$ Like the Fano resonance, our antiresonance is also due to the interference between electron path through the continuum of states (conduction band) and scattering by the extra impurity state. It is well known that Fano resonance is a universal phenomenon that can be observed in various systems. It exists in strongly correlated systems. In fact, Coulomb interaction is very often responsible to the coupling between the continuum of states and localized impurity state. This coupling, in turn, is responsible to the Fano resonance. Thus, we should also expect that our antiresonance effect is universal, and survive from the electron-electron interaction. To observe experimentally this antiresonance phenomenon, one needs to have a clean one-dimensional system with precise doping. One of such systems is probably carbon nanotubes because of great progress in its synthesis. The antiresonance could be revealed through a nonlinear current-volume $(I-V)$ measurement at low temperature. Although our antiresonance may be due to the same physical origin as that of the Fano resonance, the current phenomenon comes from the destructive interference rather than constructive one. Also, the energy dependence of the transmission coefficient is 
symmetric around antiresonance point. On the other hand, one important feature of the Fano resonance is the asymmetry of physical quantities around the resonance point.

Finally, it would not be complete if we do not point out the differences between our interpretation and that in Ref. 7 about the $a b$ initio results. The explanation of Ref. 7 relies on the special band structure of graphite, namely, zero gap semiconductor in which the Fermi surface consists of only two points of $k_{F}$ and $-k_{F}$. Obviously, our interpretation does not need to use this special property of graphite. Thus, this can be used to test experimentally the correctness of our prediction.
In summary, an antiresonance scattering can occur when an extra defect level is introduced into the conduction band of a one-dimensional system. At the antiresonance scattering, the electron transmission coefficient vanishes, leading to the disappearance of quantum conductance. This result explains well the $a b$ initio calculations ${ }^{7}$ on conductance of metallic carbon nanotubes. Although there are some similarities between this antiresonance and that of the Fano resonance, their main features are clearly different.

This work was supported by grants from the Research Grant Council of HKSAR, China. Y. Wang was also supported by NSF of China under Grant No. 19845112.
${ }^{1}$ B.J. van Wees, H. van Houten, C.W.J. Beenakker, J.W. Williamson, L.P. Kouwenhoven, D. van der Marel, and C.T. Foxon, Phys. Rev. Lett. 60, 848 (1988).

${ }^{2}$ D.A. Wharam, T.J. Thornton, R. Newbury, M. Pepper, H. Ritchie, and G.A.C. Jones, J. Phys. C 21, L209 (1988).

${ }^{3}$ N.K. Patel, J.T. Nicholls, L. Martin-Moreno, M. Pepper, J.E.F. Frost, D.A. Ritchie, and G.A.C. Jones, Phys. Rev. B 44, 13549 (1991).

${ }^{4}$ K.J. Thomas, J.T. Nicholls, M.Y. Simmons, M. Pepper, D.R.
Mace, and D.A. Ritchie, Phys. Rev. Lett. 77, 135 (1996).

${ }^{5}$ S. Iijima, Nature (London) 354, 56 (1991).

${ }^{6}$ P. Poncharal, Z.L. Wang, D. Ugarte, and W.A. de Heer, Science 283, 1513 (1999).

${ }^{7}$ H.J. Choi, J. Ihm, S.G. Louie, and M.L. Cohen, Phys. Rev. Lett. 84, 2917 (2000); P.L. McEuen, M. Bockrath, D.H. Cobden, Y.G. Yoon, and S.G. Louie, ibid. 83, 5098 (1999).

${ }^{8}$ A. Szafer and A.D. Stone, Phys. Rev. Lett. 62, 300 (1989).

${ }^{9}$ U. Fano, Phys. Rev. 124, 1866 (1961). 